\title{
Fast Acquisition for DS/FH Spread Spectrum Signals by Using Folded Sampling Digital Receiver
}

\author{
Xiaojie Wen ${ }^{1},,^{2},{ }^{3}$ \\ Beijing Institute of Satellite Information Engineering \\ Beijing, 100086, China \\ E-mail:ziwen7189@aliyun.com
}

\section{Bo Yang}

Beijing Institute of Satellite Information Engineering

Beijing, 100086, China

E-mail:bagoiybe126.com

\section{Guangnan Zou}

Beijing Institute of Satellite Information Engineering

Beijing, 100086, China

E-mail:zougnanby5030163.com

\begin{abstract}
A fast acquisition method is proposed to improve the detection speed of direct sequence/ frequency hopping spread spectrum (DS/FHSS) communication system. A novel digital wideband receiver by using folded sampling is presented to reduce the searched hopping frequencies in coarse acquisition. There are two selective filters connected with the RF downconverter in the new scheme, one of which is wideband and the other is narrow and similar as the traditional one. The acquisition is achieved on two stages: firstly, the folded sampling is used when the wideband filter is selected and the hopping frequencies on multiple Nyquist zones are folded together on the first one, then fine acquisition is performed while the narrow band filter is selected. The detection performance of the propose method is deduced through state flow graph and verified by simulation. The results show the new method performs better when compared with the traditional one. It can effectively promote the detection speed with few detection performance loss.
\end{abstract}

ISCC 2017

16-17 December, 2017

Guangzhou, China

\footnotetext{
${ }^{1}$ Speaker

${ }^{2}$ This work is supported by Natural Science Foundation of China under Grant 61401030

${ }^{3}$ Corresponding Author
} 


\section{Introduction}

DS/FHSS is the prefered communication method while LPI and LPJ capabilities are required. Fast synchronization is the essential precondition for the transceiver and the acquisition speed is essential especially when the DS/FHSS signal is transmitted in a burst manner.

The acquisition of DS/FH spread spectrum is an exciting research topic [1-8]. The search process is generally performed from two dimensions [1,2]: FH pattern and PN code phases. The full parallel method, which detects the two dimensions simultaneously, is infeasible while PN code is long for its extremely large hardware consumption [3]. The serial search tests the FH pattern and PN code phases one by one [4,5]. A two-step acquisition technique was introduced [6] to search the FH pattern at the first step and PN code phase at the secondary step. It is convenient in implementation but slow in acquisition especially when the $\mathrm{FH}$ and PN code is long. To overcome this demerit, a fast scanning and waiting method is thus provided [2]. The performance is measured with detection probability and mean acquisition time.

A folded sampling digital receiver is presented herein to further improve the search speed. In the traditional methods, the signal delivered by RF (Radio Frequency) downconverter is filtered in hopping carrier spacing [1,2,4 9] and outputs IF (Intermediate Frequency) signal before acquisition process. Whereas there are two selective filters connected with the downconverter in the new scheme, one of which is wideband and the other is narrow and similar as the traditional one. The folded sampling is used when the wideband filter is selected and the hopping frequencies on multiple Nyquist zones are folded together on the first one. Thus, the searched hopping frequencies are decreased while the noise floor is higer in acquisition process. The detection performance of the propose method is deduced and verified. The results show the proposed scheme outperforms than previous work, because it can effectively promote the detection speed.

\section{Proposed Folded Sampling Digital Receiver and Acquisition Scheme}

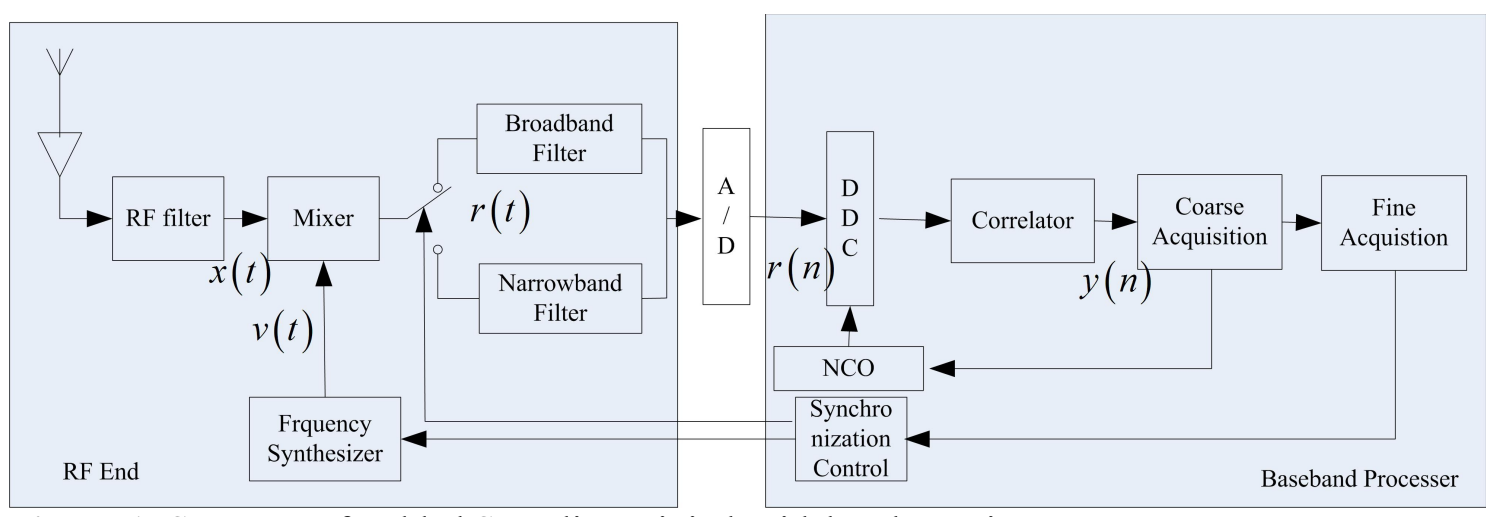

Figure 1 :Structure of Folded Sampling Digital Wideband Receiver

The receiver infrastructure of the proposed method is shown in Fig.1. Except for the power supply, the receiver is composed of RF front end, A/D converter and baseband processor.

The received signal at the RF front end of the receiver in one hop dwell is

$$
x(t)=\sqrt{2 \mathrm{~S}} c\left(t-\zeta T_{c}\right) \cos \left[2 \pi\left(f_{c}+f_{i}\right) t+\theta\right]+N(t)
$$


In (2.1), $S$ is the transmitter signal power, $c\left(t-\zeta T_{c}\right)$ is the PN sequence with length $\mathrm{L}$ and code interval $T_{c}$ seconds, $\quad \zeta T_{c}$ is the relative delay between input PN code and local one, $f_{c}$ is the receiver carrier, $f_{i}$ is the frequency of hop pattern $\left\{f_{1}, f_{2}, f_{3}, \ldots, f_{N}\right\} \quad, \quad N$ is the size of hopping pattern, $\theta$ is the phase offset, and $N(t)$ is the noise introduced by AWGN channel.

The hopping carrier spacing (hop slot) is $B_{F H}$, and the total hopping bandwidth is $B_{w}=N B_{F H}$. If digitizing the hopping signal in whole bandwidth, Nyquist sampling frequency is $2 \mathrm{~B}_{w}$. The sampling frequency in the proposed method is $f_{s}=2 \mathrm{~B} / M, M=1,2,3, \ldots$, where $M$ is folding factor. The bandwidth of broadband and narrowband filter is $B_{w} / M$ and $B_{F H}$ respectively.

The frequency synthesizer works in three modes: (1) waiting mode: output fixed frequency $f_{c}$; (2) scanning mode: hop over $\left\{f_{c}+(k-1) f_{s} / 2, k=1,2, \ldots, M\right\} \quad$; (3) normal mode: hop synchronizely with the receiver signal.

In coarse acquisition, the frequency synthesizer works in waiting mode and the mixer output is filtered through the broadband filter. From Fig. 2, it is seen that $M$ hopping frequencies $\left\{f_{j, 1}, f_{j, 2}, \ldots, f_{j, M}\right\}$ are folded together within the output frequency range $\left(0, f_{s} / 2\right)$. Thus, the hopping frequencies to be searched idecrease from $N$ to $N^{\prime}=N / M$, which can be expressed as $\left\{f_{1}, f_{2}, f_{3}, \ldots, f_{N^{\prime}}\right\}$. The FH pattern is alternated simultaneously.

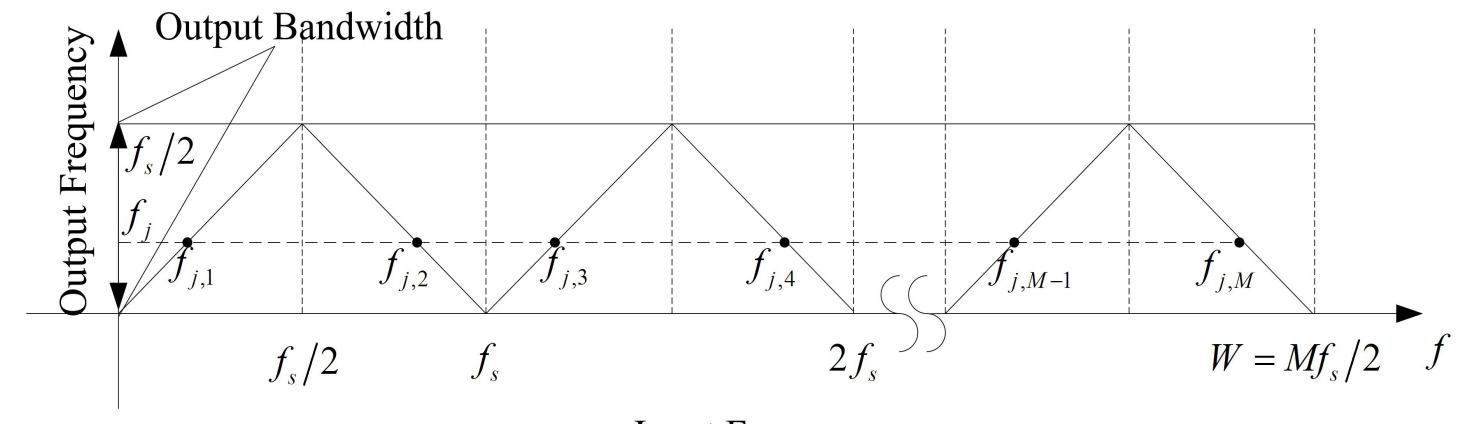

Figure 2 : The Nyquist Folded Sampling Graph

Input Frequency

The signal sampled by $\mathrm{A} / \mathrm{D}$ converter is:

$$
r(n)=\sqrt{2 \mathrm{~S}} c\left(n / f_{s}-\zeta T_{c}\right) \cos \left[2 \pi \mathrm{n}\left(f_{i_{-} \text {folded }}\right) / f_{s}+\theta\right]+N(n / f s)
$$

Where $i_{-}$folded $=1,2, \ldots, N^{\prime}$, and given by

$$
f_{i_{-} \text {folded }}=\left\{\begin{array}{l}
f_{i}-\frac{f_{s}}{2}(k-1), \text { kodd } \\
k f_{s} / 2-f_{i}, k \text { even }
\end{array}\right.
$$

The $r(n)$ is converted to baseband by the DDC (Digital Down Converter) and correlated with local PN code. The search method in coarse acquisition can be the same as traditional algorithm. Without loss of generality, a fast acquisition algorithm without any prior knowledge is used as in [2]. Let $f_{j}$ denote DDC's local oscillator's frequency, and the acquisition algorithm proposed as follows:

Step 1: set local oscillator's frequency to the first hop frequency $f_{j}=f_{1}$. 
Step 2: scan $f_{j}$ for $M T_{c}$ seconds. Find the peak of the correlation $R_{\max }$.

Step 3: if $R_{\max }>\eta$, record $f_{i_{-} \text {folded }}=f_{j}$ and go to Step 4; otherwise go to Step 5 .

Step 4: if at least $B$ of $A$ independent correlation values pass the validation, the search of input folded hop frequency declares success.

Step 5: if acquisition is failed in Step 3 or Step 4 and the hopping pattern is not searched totally ( $j<N^{\prime}$ ), then $j=j+1 \quad$ (switch to Step 2) and continue searching until the $j=N^{\prime} \quad$ (switch to step 1$)$.

After successful coarse acquisition, there are $M$ frequencies $\left\{f_{j, 1}, f_{j, 2}, \ldots, f_{j, M}\right\} \quad$ mapped to $f_{j}$. Fine acquisition is performed as follows.

The frequency synthesizer is set in scanning mode and hops over $\left\{f_{c}+(k-1) f_{s} / 2, k=1,2, \ldots M\right\}$, while the DDC's local oscillator frequency is:

$$
f_{D D C, k}=\left\{\begin{array}{c}
f_{j}, k \text { odd } \\
f_{s} / 2-f_{j}, k \text { even }
\end{array}\right.
$$

The correlation curve is obtained in every scanned hop, whose peak is preserved. Find the maximum of $M$ peaks and record the corresponding $k$. Then the exact frequency of $x(t)$ is $\left\{f_{c}+f_{j . k}\right\}$. If $M=1$, the proposed method can be seen the special case and similar to the traditional one while the fine acquisition is no longer demanded.

\section{Performance Analysis of the Proposed Method}

\subsection{Correlation Properties of Folded Sampled Signal}

The in-phase and quadrature results of correlator in Fig.1 can be expressed as :

$$
\begin{aligned}
e_{I} & =\sqrt{S} T_{c} \cos (\theta) \delta\left(f_{i_{-} \text {folded }}-f_{j}\right) \sum_{k=1}^{M} c_{p+k} c_{k}+\sum_{k=1}^{M} N_{c}(q+k) c_{k} \\
& =y \cos (\theta) \delta\left(f_{i_{-} \text {folded }}-f_{j}\right)+N_{I}
\end{aligned}
$$

And

$$
\begin{aligned}
e_{Q} & =\sqrt{S} T_{c} \sin (\theta) \delta\left(f_{i_{-} \text {folded }}-f_{j}\right) \sum_{k=1}^{M} c_{p+k} c_{k}+\sum_{k=1}^{M} N_{s}(q+k) c_{k} \\
& =y \sin (\theta) \delta\left(f_{i_{-} \text {folded }}-f_{j}\right)+N_{Q}
\end{aligned}
$$

Where $N_{I}$ and $N_{Q}$ are AWGN with $N\left(0, \sigma_{n}^{2}\right), \sigma_{n}=M N_{0} L T_{c} / 2, \delta(\bullet)$ is given by

$$
\delta(z)=\left\{\begin{array}{l}
1, z=0 \\
0, \text { else }
\end{array}\right.
$$

In (3.1) and (3.2), $\quad y \quad$ is equal to

$$
y \approx\left\{\begin{array}{l}
m_{1}=\sqrt{s} M T_{c}, H_{1} \\
m_{0}=0, H_{0}
\end{array}\right.
$$

The correlation $R=\sqrt{e_{I}^{2}+e_{Q}^{2}}$ is subject to Rice distribution :

$$
f\left(R \mid H_{i}\right)=\frac{R}{\sigma_{n}^{2}} \exp \left\{-\frac{R^{2}+m_{i}^{2}}{2 \sigma_{n}^{2}}\right\} I_{0}\left(\frac{R m_{i}}{\sigma_{n}^{2}}\right), R \geq 0, i=0,1
$$

The cumulative distribution of $R$ can be given by 


$$
F(R \mid H i)=1-Q_{1}\left(\frac{m_{i}}{\sigma_{n}}, \frac{R}{\sigma_{n}}\right)
$$

Where $Q_{1}(\bullet) \quad$ is the Marcum function.

\subsection{Mean Acquisition Time}

Assuming there is no prior knowledge of the received signal's hopping frequency, in the new scheme, there are $N^{\prime}=N / M$ hopping frequencies to be searched at one acquisition process. The mean acquisition time can be obtained with the state flow diagram [10,11].

In Fig. 3, the state $\left\{S_{1}, S_{2}, S_{3}, \ldots, S_{N^{\prime}}\right\} \quad$ corresponds to the tested $N^{\prime}$ hopping frequencies. State $S_{N^{\prime}}$ is the state the input folded signal matches the local hopping frequecncy and the successful acquistion is possible, and the false alarm state ( $F A$ ) is a contrary state. 'Coarse Acq' and 'Fine Acq' represent the folded received signal that is detected and the unfolded received signal is searched respectively.

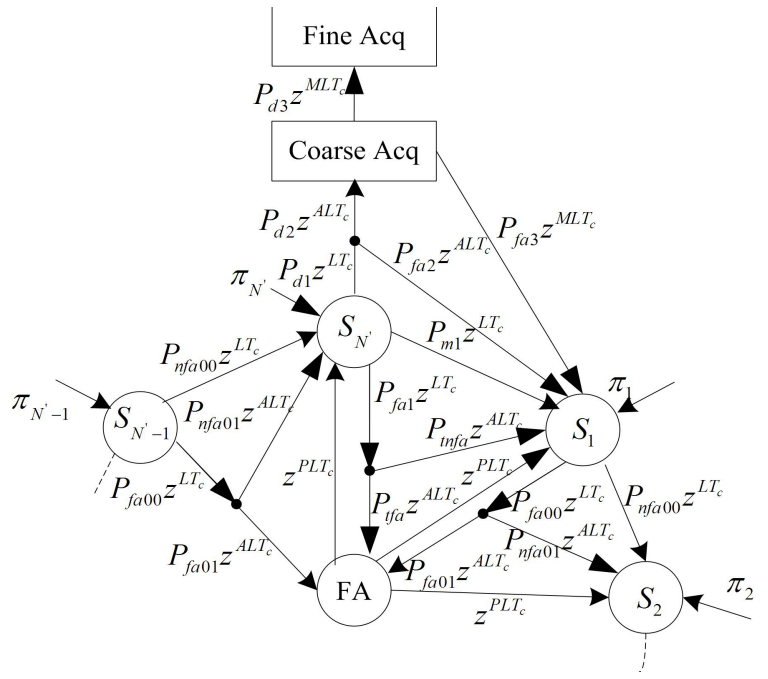

Figure 3 : State Flow Diagram of the Acquisition with A Priori Distribution $\quad\left\{\pi_{j}\right\}$

In 'Coarse Acq' process, the detection is completed when the input folded hop frequency is searched successfully, and the probability is given by :

$$
P_{d l}=\int_{\eta}^{\infty} f\left(x \mid H_{1}\right)\left(F\left(x \mid H_{0}\right)\right)^{L-1} d x
$$

The missing probability in State $S_{N^{\prime}}$ corresponding to that none of the correlations exceed the detection threshold:

$$
P_{m l}=F\left(\eta \mid H_{1}\right)\left(F\left(\eta \mid H_{0}\right)\right)^{L-1}
$$

If the searched hop frequency is incorrect, the state switch from $S_{N^{\prime}}$ to $F A$ and the probability is

$$
P_{f a l}=1-P_{d l}-P_{m l}
$$

The detection probability and the false alarm probability in checking can be expressed as cumulative binomial distribution

$$
P_{d 2}=\sum_{n=B}^{A}\left(\begin{array}{l}
A \\
n
\end{array}\right) P_{d}{ }^{n}\left(1-P_{d}\right)^{A-n}
$$




$$
P_{t f a}=\sum_{n=B}^{A}\left(\begin{array}{l}
A \\
n
\end{array}\right) P_{f a}^{n}\left(1-P_{f a}\right)^{A-n}
$$

Where

$$
\begin{gathered}
P_{d}=\int_{0}^{\infty} f\left(x \mid H_{1}\right)\left(F\left(x \mid H_{0}\right)\right)^{L-1} d x \\
P_{f a}=\int_{0}^{\infty} f\left(x \mid H_{0}\right) F\left(x \mid H_{1}\right)\left(F\left(x \mid H_{0}\right)\right)^{L-2} d x
\end{gathered}
$$

The $P_{n f a 0}$ and $P_{n f a l}$ corresponding to $\left\{S_{1}, S_{2}, \ldots S_{N^{\prime}-1}\right\}$ is obtained as follows:

$$
\begin{aligned}
P_{n f a 0} & =\left(F\left(\eta \mid H_{0}\right)\right)^{L} \\
P_{n f a l} & =1-\sum_{n=B}^{A}\left(\begin{array}{l}
A \\
n
\end{array}\right) P_{f a 0}{ }^{n}\left(1-P_{f a 0}\right)^{A-n}
\end{aligned}
$$

Where $P_{n f a 0}=\left(F\left(\eta \mid H_{0}\right)\right)^{M}$.

In "Fine Acq" process, $\quad M$ frequencies are searched and the detection probability can be calculated by

$$
P_{d 3}=\int_{0}^{\infty} f\left(x \mid H_{1}\right)\left(F\left(x \mid H_{0}\right)\right)^{M-1} d x
$$

The false alarm probability is

$$
P_{f a 3}=1-P_{d 3}
$$

When the priori distribution $\left\{\pi_{j}\right\} \quad$ is uniform, the generating function is

$$
P_{A C Q}(z)=\frac{H_{D}(z)\left(1-H_{0}{ }^{N^{\prime}}(z)\right)}{N^{\prime}\left(1-H_{M}(z) H_{0}^{N^{\prime}-1}(z)\right)\left(1-H_{0}(z)\right)}
$$

Where $H_{D}(z)$ represents the transfer function from state $S_{N^{\prime}}$ to "Coarse Acquisition", $H_{M}(z)$ represents the transfer function connecting $S_{N^{\prime}}$ with $S_{1}$ while $H_{0}(z)$ represents the transfer function between $\left(S_{i}, S_{i+1}\right) ; i=1, \ldots, N^{\prime}-1$.

They are obtained as follows:

$$
\begin{gathered}
H_{D}(z)=P_{d 1} z^{L T_{c}} P_{d 2} z^{A L T_{c}} P_{d 3} z^{M L T_{c}} \\
H_{M}(z)=P_{m 1} z^{L T_{c}}+P_{d 1} z^{L T_{c}} P_{f a 2} z^{A L T_{c}}+P_{f a 1} z^{L T_{c}}\left(P_{t n f a} z^{A L T_{c}}+P_{t f a} z^{A L T_{c}} z^{P L T_{c}}\right) \\
+P_{d 1} z^{L T_{c}} P_{d 2} z^{A L T_{c}} P_{f a 3} z^{M L T_{c}} \\
H_{0}(z)=P_{n f a 0} z^{L T_{c}}+P_{f a 00} z^{L T_{c}}\left[P_{n f a 01} z^{A L T_{c}}+P_{f a 01} z z^{(A+P) L T_{c}}\right]
\end{gathered}
$$

The mean acquisition time is:

$$
\begin{gathered}
E\left(T_{A C Q}\right)=\left.\frac{\mathrm{d} P_{A C Q}(z)}{\mathrm{d} z}\right|_{z=1} \\
=\frac{L T_{c}}{P_{d 1} P_{d 2} P_{d 3}}\left\{1+A\left(P_{d 1}+P_{f a l}\right)+P P_{t f a} P_{f a l}+M P_{d l} P_{d 2}+\frac{\left(N^{\prime}-1\right)}{2} P_{0}\right\}
\end{gathered}
$$

Where $P_{0}=\left[1+A P_{f a 00}+P P_{f a 00} P_{f a 01}\right]\left(2-P_{d 1} P_{d 2} P_{d 3}\right)$ 


\section{Results and Discussion}

In this Section, the detection performance of the proposed algorithm is evaluated by simulation. A traditional one is adopted for comparison, where a fast PN code phase scanning and serial search for FH pattern method [2] is adopted as a comparison. In evaluating the performance, the following parameters were considered: PN code rate $1 \times 10^{6} \mathrm{chip} / \mathrm{s}$ and length $L=64$, number of hopping frequencies $N=64$, hoping frequency set

$$
\begin{aligned}
& \left\{f_{1}, f_{2}, f_{3}, \ldots, f_{64}\right\}=\{1,3,5, \ldots, 127\} \mathrm{MHz}, \text { verification pair } A=3, B=2 \\
& P=100 .
\end{aligned}
$$

Fig. 4 shows the correlation curves for two acquisition methods with the received signal $\mathrm{SNR}=5 \mathrm{~dB}$ and the folding factor $M=2$ in the proposed method. The local oscillator's frequency $f_{j}=17 \mathrm{MHz}$. When the hopping frequency $f_{i}=17 \mathrm{MHz}$ and $f_{i}=111$ $\mathrm{MHz}$, the correlation peak by using proposed method can still be achieved in Fig. 5.

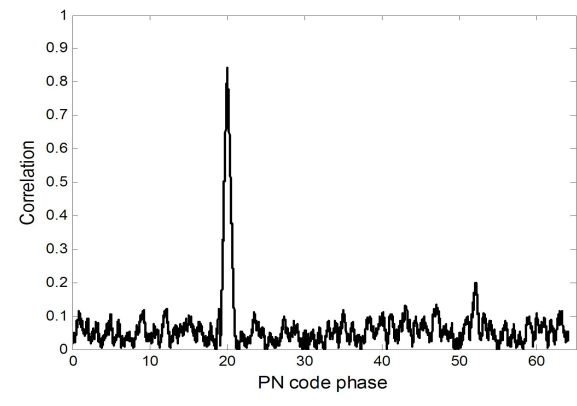

(a) Traditional method

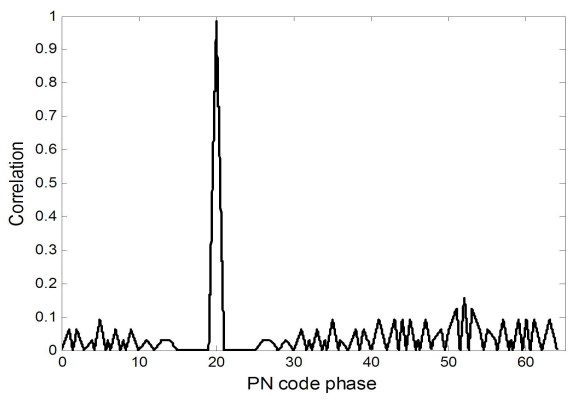

(b) Proposed Method

Figure 4 : Correlation Curves for Two Methods when $\quad M=2, \quad f_{i}=17 \mathrm{MHz}$ and $f_{j}=17$ $\mathrm{MHz}$

The detection performances of the traditional method and the proposed scheme with different folding factor $M$ are presented in Fig. 6. The hopping frequency and the local oscillator's frequency are the same here, while the situation $f_{i} \neq f_{j}$ is not discussed as the traditional method that cannot detect signal. The detection of folded hop frequency and the accurate one declared success once the single correlation peak exceeds the threshold. The false alarm probability is $10^{-5}$ here. It is seen that there is a loss of detection performance of hopping frequency and spread code after folded sampling. When the folding factor grows, the performance degradation becomes severe due to the noise floor increases while the folded sampling is applied.

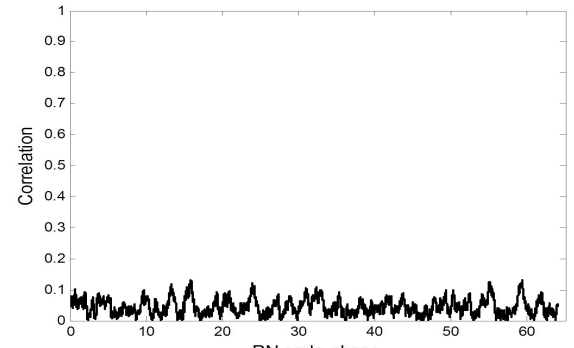

(a) Traditional method

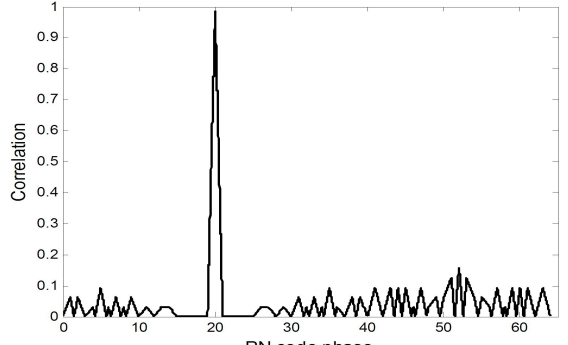

PN code phase
(b) Proposed Method

Figure 5 : Correlation Curves for Two Methods When $M=2, \quad f_{i}=111 \mathrm{MHz}$ and $f_{j}=17$ $\mathrm{MHz}$

In Fig. 7 the detection speed is compared between the proposed method and the traditional one. The threshold $\eta$ is selected to maximize the detection speed. In the new algorithm, the 
unmatched hopping frequencies are excluded quickly and searched hopping frequencies are decreased, while the detection performance suffers degradation. While the SNR is high and the dectction performance has smaller effects than the tested hopping frequencies, the proposed algotithm can effectively promote the detection speed and outperform the traditional method. The proposed method with $\quad M=2$ costs about $50 \%$ of the traditional acquisition time and $M=4$ costs about $25 \%$ respectively.

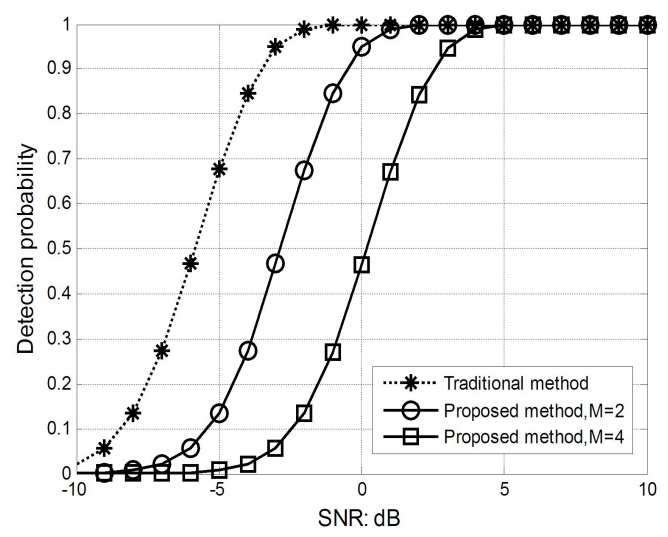

Figure 6 :Detection Probability of Two Methods Methods

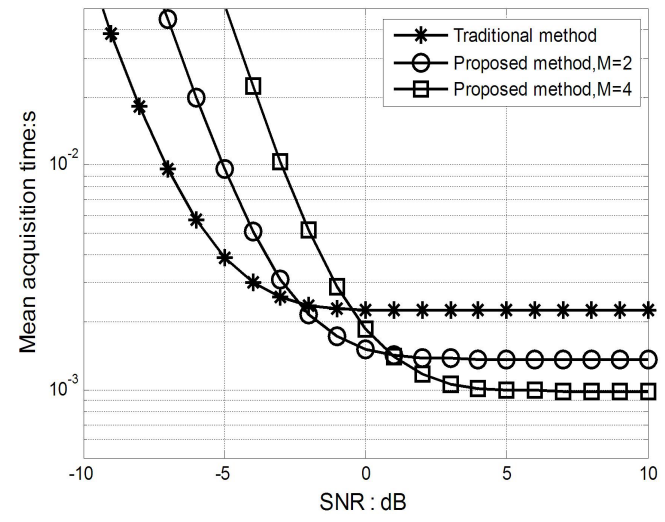

Figure 7 : Mean Acquisition Time of Two

\section{Conclusions}

In this paper, the folded sampling digital receiver scheme to decrease the acquisition time of DS/FHSS communication system has been proposed. The detection performance of the propose method is deduced through the state flow graph and verified by simualtion. The numerical results show the new method performs better when compared with the traditional one when the SNR is high, it can effectively promote the detection speed with few detection performance loss.

\section{References}

[1] W. Yang, S. Meng, J. Wang, J. Liu. Acquisition performance analysis of a synchronization scheme of DS/FH hybrid spread spectrum signals for $T T \& C[C]$. Electronic Measurement \& Instruments, 2009. Beijing: 4395-4399.

[2] X. Wen, W. Song, Y. Zhang, D. Shao and S. Li, Performance Analysis of a Digital Acquisition Circuit for DS/FH Spread Spectrum Signal[C]. 2009 International Symposium on Computer Network and Multimedia Technology, Wuhan: 1-4.

[3] X. Gou, B. Zhang, Q. Wu, et al. A Fast Synchronization Method of DS/FH Hybrid Spread Spectrum TT\&C System[J]. Energy Procedia, 2011, 13:8493-8502.

[4] J. Chen, X. Zhou. A Study of Sychronization and anti-jamming for DS/FH Hybrid Spread Spectrum Signal[D]. University of Electronic Science and Technology of China. May.2009.(InChinese)

[5] N. Benvenuto, G. Guidotti and S. Pupolin. Performance of a digital acquisition circuit for hybrid FH-DS spread spectrum systems[C] Military Communications Conference, 1988: 971-975.

[6] F. Wang; D. Zhou. Cascade connection serial parallel hybrid acquisition synchronization for DSFHSS in air-ground data link $[\mathrm{C}]$.Second International Conference on Space Information Technology 2007. 
[7] W. Zhan, X. Zhang. Design of Baseband Signal Processing in TT\&C System and Hardware Realization Based on DSSS/FHSS Technique. International Conference on Intelligent System Design and Engineering Application.IEEE Computer Society 2010: 560-564.

[8] F. J. Block and H. Nguyen. Packet acquisition for low-complexity frequency-hop receivers. MILCOM 2008, San Diego, CA, 2008:1-6

[9] L. Simone, N. Salerno, M. Maffei. Frequency-Hopping Techniques for Secure Satellite TT\&C: System Analysis \& Trade-Offs[C] International Workshop on Satellite and Space Communications. IEEE, 2006:13-17.

[10] A. Polydoros and C. Weber. A Unified Approach to Serial Search Spread-Spectrum Code Acquisition--part I: General Theory[J]. IEEE Transactions on Communications, 1984, 32(5): $542-$ 549.

[11] H. Li, M. Lu and Z. Feng. Improved Zero-Padding Method for Rapid Long PN-Code Acquisition [J]. IEEE Transactions on Signal Processing, 2008, 56(8): 3795-3799 